\title{
Relationship Between Incremental Glucose Peak and Diabetic Retinopathy in Tertiary Hospital: Liaoning Diabetic Microvascular Complications Study (LD-MCS)
}

\section{Xiang Li}

China Medical University

\section{Litong Yao}

Department of Breast Surgery, the First Affiliated Hospital of China Medical University

\section{Xinyu Wang}

China Medical University

\section{Yifan Zhong}

First Affiliated Hospital of China Medical University

\section{Yan Wang}

General Hospital of Liaohe Oil Field

\section{Jingyang Wu}

First Affiliated Hospital of China Medical University

Jin Geng

First Affiliated Hospital of China Medical University

\section{Yun Zhou}

First Affiliated Hospital of China Medical University

Jiahua Zhang

First Affiliated Hospital of China Medical University

Jun Chen

First Affiliated Hospital of China Medical University

\section{Yingying Xu}

First Affiliated Hospital of China Medical University

\section{Lei Chen}

First Affiliated Hospital of China Medical University

Lei Liu ( $\square$ liuleijiao@163.com )

First Affiliated Hospital of China Medical University

\section{Yudong Hu}

First Affiliated Hospital of China Medical University 
Original investigation

Keywords: diabetic retinopathy; incremental glucose peak; glycemic variability; oral glucose tolerance test; Liaoning Diabetic Microvascular Complications Study

Posted Date: October 19th, 2020

DOI: https://doi.org/10.21203/rs.3.rs-91509/v1

License: (c) (i) This work is licensed under a Creative Commons Attribution 4.0 International License.

Read Full License 


\section{Abstract}

Background: Glucose variability may contribute to macrovascular complications of diabetes but evidence for its association with diabetic retinopathy (DR) is limited. This study is aimed to investigate the correlation between incremental glucose peak (IGP) and diabetic retinopathy (DR) in Chinese population with type 2 diabetes.

Methods: A hospital-registry, case-control study collecting data from Liaoning Diabetic Microvascular Complications Study (LD-MCS) during 2012-2018. 795 adults with DR were included and matched with two diabetic controls per case $(n=1590)$ by gender and age. IGP was defined as the peak minus baseline plasma glucose value during 5-point oral glucose tolerance test (OGTT). DR was diagnosed by two-field fundus photographs under Early Treatment Diabetic Retinopathy Study criteria and stratified into mild non-proliferative DR (NPDR), moderate NPDR, and vision-threatening DR (VTDR). Logistic regression analysis was applied to determine the relationships.

Results: Among 2385 participants (age, $58.40 \pm 11.52$ years; $57.11 \%$ men), higher IGP was independently associated with the presence of any DR (odds ratio [OR], 1.05; 95\% confidence interval [CI], 1.02-1.07; $P<$ 0.001), moderate NPDR (OR, 1.06; 95\% $\mathrm{Cl}, 1.01-1.12 ; P=0.020)$ and VTDR (OR, 1.13; $95 \% \mathrm{Cl}, 1.07-1.19 ; P<$ 0.001), respectively. Moreover, estimated ratio for any DR and VTDR from low-quartile IPG increased by $1.33(95 \% \mathrm{Cl}, 1.04-1.70 ; P$ for trend $=0.021)$ and $3.64(95 \% \mathrm{Cl}, 1.97-6.72$; $P$ for trend $<0.001)$, respectively.

Conclusions: OGTT-derived IGP was independently associated with DR severity scales in Chinese patients with type 2 diabetes. More attention by health care personnel needs to be paid to glucose variability of this population.

\section{Background}

Diabetic retinopathy (DR), as one of the common complications of diabetes mellitus, is characterized by microangiopathic alterations at the fundus. $[1,2]$ It was estimated worldwide that the prevalence of DR and vision-threatening stage of DR was up to $34.6 \%$ and $10.2 \%$, respectively.[3] The number of people affected by type 2 diabetes increases worldwide, with associated increases in its complications .[4] Moreover, as the most frequent cause of vision loss across many countries, ${ }^{[2]}$ impaired vision triggered by DR imposed much burden on public health care resources.[5]

Great strides in the epidemiological investigations of DR, which confirmed that consistent modifiable risk factors for DR included hyperglycemia.[1, 6] Intensive glycemic control reported by the United Kingdom Prospective Diabetes Study (UKPDS) and the Diabetes Control and Complications Trial (DCCT) was proven to be beneficial of prevention of the incidence and progression of DR, and the UKPDS also reported that every $10 \%$ decrease of glycated hemoglobin $\mathrm{A} 1 \mathrm{c}(\mathrm{HbA1c})$ was accompanied with one quarter decreased danger of microangiopathy for type 2 diabetes, including DR. $[7,8]$ The metabolic pathways initiated by hyperglycemia may explain this association.[9] 
Glycemic variability (GV), the dynamic evaluation of plasma glucose changes, deepened the concept of glycemic control beyond $\mathrm{HbA} 1 \mathrm{c}$. However, evidence for the effect of GV on retinopathy of type 2 diabetes was limited. Sonoda et al. suggested the mean amplitude of glycemic excursions (MAGE) had no significant correlations with DR.[10] However, some evidences certified direct or indirect damage to retina caused by GV based on 72-hour continuous glucose monitoring system (CGM) or 1,5-anhydroglucitol (an indicator related to postprandial hyperglycemia and GV), especially in acute fluctuation and hyperglycemia.[11-13]

Clinically, CGM use is still considered experimental and limited, we attempted to focus on incremental glucose peak (IGP) derived from oral glucose tolerance test (OGTT), which had be considered as an appropriate alternative for approximation of GV.[14, 15] IGP refers to the glycemic difference between baseline and peak during OGTT, which has been found with significant associations with some macrovascular events. $[16,17]$ However, its association with microvascular complications, such as DR, has not been explored yet. Accordingly, this study aimed to investigate relationships between IGP and DR as well as its severity strata in Chinese population with type 2 diabetes.

\section{Methods}

\section{Research design and study population}

We used data from Liaoning Diabetic Microvascular Complications Study (LD-MCS). The LD-MCS is a series of hospital-based observational studies and aimed to identify risk factors and biomarkers of diabetic microvascular complications for Chinese adults with type 2 diabetes. Theory basis and methodology of the LD-MCS were reported in the Appendix (see Additional file 1).

Cases and controls defined as participants with DR or without DR, respectively. The control group sample was calculated by power of $80 \%$, ratio of cases to controls: $1: 2$ and percentage of controls exposed to higher GV: $10 \%$ and Odds ratio 1.20 (both provided based on pilot investigation). Added $10 \%$ for losses, sample calculation resulted in 620 cases and 1240 controls.

After assessment in LD-MCS, 1469 cases with DR were collected from the database and subsequently some participants with incomplete data, either because an OGTT contraindication ( $n=568$; i.e. plasma glucose levels $>11.0 \mathrm{mmol} / \mathrm{L}$ before initiation of the OGTT), or ambiguous fundus grades $(n=106)$ were removed. Each remaining case was matched randomly with two controls without any history of DR by same gender and age in the database. Finally, 795 confirmed cases (54.1\%) with DR and 1590 corresponding controls amounting to 2385 participants were recruited in this study.

\section{Assessment Of Diabetic Retinopathy}

Two ophthalmologists of the LD-MCS were invited to independently evaluate fundus situation of participants and confirm the diagnosis and severity scales of DR under guidelines of the Early Treatment 
for Diabetic Retinopathy Study (ETDRS).[18] Each image was a two-field colorful one taken by nonmydriatic digital retinal photography (CR6-45NM; Canon, Inc., Tokyo, Japan). The diagnosis of DR was ascertained by the following signature lesions: fundus microaneurysms, hemorrhage, cotton wool spots, intraretinal microvascular impairments, hard exudates, venous beading and new vascularization. Next, retinopathy was scored into five grades: . non-DR; $邓$. mild non-proliferative DR (NPDR) (ETDRS stage 20);

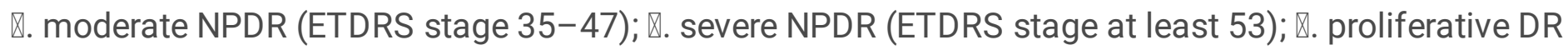
(PDR) (with abnormal growth of definite new vessels).[19] Similarly, retinopathy was also divided into two categories, including none vision-threatening DR (non-VTDR) and VTDR which was certified by clinically significant maculopathy, severe NPDR or PDR.[18] The grade outcomes depended on the severity of the worse eye. In case of disagreement with the diagnosis, a third ophthalmologist would attend.

\section{Assessment Of G Incremental Glucose Peak And Covariates}

We performed comprehensive medical assessment of health profiles of each participant and collected their demographic, anthropometric information and clinical examination results on admission date. Gender, age, smoker, drinker, use of antidiabetic agents, duration of diabetes, height, weight, body mass index (BMI), heart rate (HR), blood pressures (systolic blood pressure [SBP], diastolic blood pressure [DBP] and pulse pressure [PP]) were obtained from hospital-based diabetes registry. All participants underwent a standardized 3-h $75 \mathrm{~g}$ OGTT after fasting overnight. The concentration of plasma glucose at baseline, $30 \mathrm{~min}, 60 \mathrm{~min}, 120 \mathrm{~min}, 180 \mathrm{~min}$ as fasting plasma glucose (FPG), 30-min plasma glucose (30-min PG), 60-min PG, 120-min PG, and 180-min PG, respectively. Plasma insulin levels of the five time points were processed. The peak value of post-load plasma glucose was the maximum of above glycemic concentration. IGP was the result of peak value minus FPG. Furthermore, biochemical variables included serum glycosylated haemoglobin ( $\mathrm{HbA} 1 \mathrm{c})$, and serum creatinine. Besides, we included two calculative indices, the insulin resistance index (HOMA-IR, HOMA-IR = FPG * Insulin [baseline] / 22.5) and the $\beta$-cell function (HOMA- $\beta, \mathrm{HOMA}-\beta=20$ * Insulin [baseline] / [FPG - 3.5]), in which FPG was expressed in $\mathrm{mmol} / \mathrm{L}$ and insulin was expressed in $\mu \mathrm{IU} / \mathrm{mL}$, to evaluate insulin resistance and pancreatic $\beta$-cell function.[20] Estimated glomerular filtration rate (eGFR) was calculated by the equation of Chronic Kidney Disease Epidemiology Collaboration (CKD-EPI) to evaluate renal function.[21]

\section{Statistical analysis}

All statistical analyses were performed by the SPSS version 24.0. (IBM for Windows). Continuous and categorical data were expressed as mean and SD (mean \pm SD) and percentage (No. [\%]), respectively. Data was verified for the normal distribution by Kolmogorov-Smirnov test. The Chi-square test and Fisher exact test was applied for discontinuous data comparison and the independent Student's t-test was applied for continuous variables comparison across different study population groups. One-way ANOVA test and the Chi-square test were used to compare variables between various subgroups. Three logistic regression models adjusted for confounding factors were carried out to estimate the odds ratio (OR) with a $95 \%$ confidence interval $(95 \% \mathrm{CI})$. IGP and HOMA-IR were transformed into categorical data by quartiles 
which were segregated by the 25th, 50th and 75th percentage as Quartile 1 (Q1), Q2, Q3, and Q4. Multiple R-squared liner regression analysis was performed to reveal the correlations between variables and IGP quartiles. In all analyses, $P$ value less than 0.05 was defined as statistical significance.

\section{Results}

\section{Study population characteristics}

The characteristics of 2385 participants stratified by the presence and severity of DR were presented in Table 1. Among all participants (age, $58.40 \pm 11.52$ years; $57.11 \%$ men), 795 (33.3\%) had retinopathy (533 mild NPDR [67.0\%], 140 moderate NPDR [17.6\%], and 122 VTDR [15.3\%]). Compared to non-DR group (1590 [66.7\%]), subjects with DR were like to be with longer duration of diabetes, and had higher level of weight, HR, SBP, PP, HbA1c, 120-min PG, 180-min PG, post-load plasma glucose peak, and IGP (all $P<$ 0.05). Blood glucose variability before and after OGTT among participants with different DR severity scales were described on Figure S1 in Additional file 2. Along with the advanced retinopathy condition, HbA1c, 120-min PG, 180-min PG, post-load plasma glucose peak, and IGP showed an upward tendency (HbA1c: $P=0.014 ; 180-m i n$ PG: $P=0.002$; all others: $P<0.001$ ). 
Table 1

Characteristics of included participants stratified by the presence and severity of DR

\begin{tabular}{|c|c|c|c|c|c|c|c|c|}
\hline \multirow[t]{2}{*}{ Characteristics } & \multirow{2}{*}{$\begin{array}{l}\text { Total } \\
(n= \\
2385)\end{array}$} & \multicolumn{3}{|c|}{ Presence of DR } & \multicolumn{4}{|c|}{ Severity of DR } \\
\hline & & $\begin{array}{l}\text { Non-DR } \\
(n= \\
1590)\end{array}$ & $\begin{array}{l}\text { DR } \\
(n= \\
795)\end{array}$ & $\begin{array}{l}P \\
\text { Value }\end{array}$ & $\begin{array}{l}\text { Mild } \\
\text { NPDR } \\
(n= \\
533)\end{array}$ & $\begin{array}{l}\text { Moderate } \\
\text { NPDR } \\
(n=140)\end{array}$ & $\begin{array}{l}\text { VTDR } \\
(n= \\
122)\end{array}$ & $\begin{array}{l}P \\
\text { Value }\end{array}$ \\
\hline Male, No. (\%) & $\begin{array}{l}1362 \\
(57.11)\end{array}$ & $\begin{array}{l}908 \\
(57.11)\end{array}$ & $\begin{array}{l}454 \\
(57.11)\end{array}$ & 1.000 & $\begin{array}{l}313 \\
(58.72)\end{array}$ & $\begin{array}{l}72 \\
(51.43)\end{array}$ & $\begin{array}{l}69 \\
(56.56)\end{array}$ & 0.489 \\
\hline Age, years & $\begin{array}{l}58.40 \\
\pm 11.52\end{array}$ & $\begin{array}{l}58.40 \\
\pm 11.52\end{array}$ & $\begin{array}{l}58.40 \\
\pm 11.53\end{array}$ & 1.000 & $\begin{array}{l}58.29 \\
\pm 11.54\end{array}$ & $\begin{array}{l}59.41 \pm \\
10.96\end{array}$ & $\begin{array}{l}57.71 \\
\pm 12.13\end{array}$ & 0.667 \\
\hline Height, cm & $\begin{array}{l}166.31 \\
\pm 8.19\end{array}$ & $\begin{array}{l}166.11 \\
\pm 8.28\end{array}$ & $\begin{array}{l}166.70 \\
\pm 7.98\end{array}$ & 0.095 & $\begin{array}{l}167.07 \\
\pm 7.86\end{array}$ & $\begin{array}{l}165.59 \pm \\
8.05\end{array}$ & $\begin{array}{l}166.37 \\
\pm 8.33\end{array}$ & 0.083 \\
\hline Weight, kg & $\begin{array}{l}68.26 \\
\pm 12.20\end{array}$ & $\begin{array}{l}67.88 \\
\pm 12.40\end{array}$ & $\begin{array}{l}69.03 \\
\pm 11.75\end{array}$ & 0.023 & $\begin{array}{l}69.82 \\
\pm 11.88\end{array}$ & $\begin{array}{l}67.23 \pm \\
11.56\end{array}$ & $\begin{array}{l}67.59 \\
\pm 11.11\end{array}$ & 0.086 \\
\hline $\mathrm{BMI}, \mathrm{kg} / \mathrm{m}^{2}$ & $\begin{array}{l}24.58 \\
\pm 3.29\end{array}$ & $\begin{array}{l}24.49 \\
\pm 3.30\end{array}$ & $\begin{array}{l}24.76 \\
\pm 3.26\end{array}$ & 0.061 & $\begin{array}{l}24.93 \\
\pm 3.28\end{array}$ & $\begin{array}{l}24.45 \pm \\
3.32\end{array}$ & $\begin{array}{l}24.36 \\
\pm 3.10\end{array}$ & 0.046 \\
\hline $\mathrm{HR}, \mathrm{bmp}$ & $\begin{array}{l}80.21 \\
\pm 10.78\end{array}$ & $\begin{array}{l}79.82 \\
\pm 10.49\end{array}$ & $\begin{array}{l}80.97 \\
\pm 11.31\end{array}$ & 0.026 & $\begin{array}{l}80.65 \\
\pm 11.14\end{array}$ & $\begin{array}{l}81.50 \pm \\
12.02\end{array}$ & $\begin{array}{l}81.78 \\
\pm 11.22\end{array}$ & 0.057 \\
\hline $\mathrm{SBP}, \mathrm{mmHg}$ & $\begin{array}{l}135.33 \\
\pm 18.69\end{array}$ & $\begin{array}{l}134.17 \\
\pm 17.88\end{array}$ & $\begin{array}{l}137.65 \\
\pm 20.03\end{array}$ & $<.001$ & $\begin{array}{l}137.85 \\
\pm 19.63\end{array}$ & $\begin{array}{l}137.06 \pm \\
20.41\end{array}$ & $\begin{array}{l}137.50 \\
\pm 21.44\end{array}$ & $\begin{array}{l}< \\
0.001\end{array}$ \\
\hline $\mathrm{DBP}, \mathrm{mmHg}$ & $\begin{array}{l}82.39 \\
\pm 11.08\end{array}$ & $\begin{array}{l}82.36 \\
\pm 10.86\end{array}$ & $\begin{array}{l}82.44 \\
\pm 11.50\end{array}$ & 0.871 & $\begin{array}{l}82.49 \\
\pm 11.28\end{array}$ & $\begin{array}{l}82.00 \pm \\
11.56\end{array}$ & $\begin{array}{l}82.75 \\
\pm 12.46\end{array}$ & 0.950 \\
\hline $\mathrm{PP}, \mathrm{mmHg}$ & $\begin{array}{l}52.94 \\
\pm 15.51\end{array}$ & $\begin{array}{l}51.81 \\
\pm 14.75\end{array}$ & $\begin{array}{l}55.21 \\
\pm 16.72\end{array}$ & $<0.001$ & $\begin{array}{l}55.36 \\
\pm 16.37\end{array}$ & $\begin{array}{l}55.06 \pm \\
18.34\end{array}$ & $\begin{array}{l}54.75 \\
\pm 16.40\end{array}$ & $<.001$ \\
\hline Smoker, No. (\%) & $\begin{array}{l}666 \\
(27.92)\end{array}$ & $\begin{array}{l}445 \\
(27.99)\end{array}$ & $\begin{array}{l}221 \\
(27.80)\end{array}$ & 0.923 & $\begin{array}{l}139 \\
(26.08)\end{array}$ & $\begin{array}{l}36 \\
(25.71)\end{array}$ & $\begin{array}{l}46 \\
(37.70)\end{array}$ & 0.071 \\
\hline Drinker, No. (\%) & $\begin{array}{l}509 \\
(21.34)\end{array}$ & $\begin{array}{l}332 \\
(20.88)\end{array}$ & $\begin{array}{l}177 \\
(22.26)\end{array}$ & 0.437 & $\begin{array}{l}107 \\
(20.08)\end{array}$ & $\begin{array}{l}33 \\
(23.57)\end{array}$ & $\begin{array}{l}37 \\
(30.33)\end{array}$ & 0.072 \\
\hline $\begin{array}{l}\text { Antidiabetic } \\
\text { agents, yes, No. } \\
\text { [\%] }\end{array}$ & $\begin{array}{l}2225 \\
(93.29)\end{array}$ & $\begin{array}{l}1473 \\
(92.64)\end{array}$ & $\begin{array}{l}752 \\
(94.59)\end{array}$ & 0.073 & $\begin{array}{l}514 \\
(96.44)\end{array}$ & $\begin{array}{l}127 \\
(90.71)\end{array}$ & $\begin{array}{l}111 \\
(90.98)\end{array}$ & 0.007 \\
\hline $\begin{array}{l}\text { Diabetes } \\
\text { duration, year }\end{array}$ & $\begin{array}{l}8.49 \pm \\
6.97\end{array}$ & $\begin{array}{l}8.11 \pm \\
6.87\end{array}$ & $\begin{array}{l}9.24 \pm \\
7.12\end{array}$ & $<.001$ & $\begin{array}{l}9.07 \pm \\
6.97\end{array}$ & $\begin{array}{l}10.52 \pm \\
7.77\end{array}$ & $\begin{array}{l}8.51 \pm \\
6.85\end{array}$ & $<.001$ \\
\hline
\end{tabular}

In view of confounders, Fig. 1 presented bivariate and multivariate logistic regressive analysis performed to further explore the relationships between IGP and DR as well as its severity strata. Adjusted for gender and age (model 1), IGP levels had a statistically significant correlation with the presence of DR (OR, 1.05; 
95\% $\mathrm{Cl}, 1.02-1.07 ; P<0.001)$, moderate NPDR (OR, 1.06; $95 \% \mathrm{Cl}, 1.01-1.12 ; P=0.020)$ and VTDR (OR, $1.13 ; 95 \% \mathrm{Cl}, 1.07-1.19 ; P<0.001)$, respectively. Model 2 with adjustment for variables related to severity of DR and model 3 with additional potential adjustment for FPG, HOMA-IR, serum creatinine and eGFR showed similar relationships.

When study participants were divided into four groups by IGP (Q1: IGP < 7.14; Q2: 7.14-9.42; Q3: 9.4311.81; Q4: IGP $\geq 11.82$ ) in Table 2, all glycemic parameters, including HbA1c, FPG, post-load plasma glucose concentration, and HOMA-IR had strong correlations with IGP quartiles (FPG: $P=0.002$; others: $P$ $<0.001)$. Post-load glucose levels climbed dramatically with IGP quartiles progression, while HOMA-IR descended. 
Table 2

Characteristics of included participants by quartiles of IGP

\begin{tabular}{|c|c|c|c|c|c|}
\hline \multirow[t]{2}{*}{ Characteristics } & \multicolumn{4}{|l|}{ IGP quartiles } & \multirow{2}{*}{$\begin{array}{l}P \\
\text { value }\end{array}$} \\
\hline & $\begin{array}{l}\text { Q1 (<7.14, } \\
n=600)\end{array}$ & $\begin{array}{l}\text { Q2 (7.14-9.42, } \\
n=593)\end{array}$ & $\begin{array}{l}\text { Q3 (9.43-11.81, } \\
n=596)\end{array}$ & $\begin{array}{l}\text { Q4 ( }(\geq 11.82 \\
n=596)\end{array}$ & \\
\hline Male, No. (\%) & 338 (56.33) & 340 (57.34) & 348 (58.39) & $336(56.38)$ & 0.876 \\
\hline Age, years & $\begin{array}{l}58.12 \pm \\
11.01\end{array}$ & $58.23 \pm 11.60$ & $58.67 \pm 11.46$ & $58.57 \pm 12.01$ & 0.813 \\
\hline Height, cm & $\begin{array}{l}166.01 \pm \\
8.10\end{array}$ & $166.46 \pm 8.50$ & $166.83 \pm 7.90$ & $165.93 \pm 8.22$ & 0.199 \\
\hline Weight, kg & $\begin{array}{l}68.08 \pm \\
12.12\end{array}$ & $67.95 \pm 12.62$ & $68.63 \pm 11.89$ & $68.38 \pm 12.18$ & 0.772 \\
\hline $\mathrm{BMI}, \mathrm{kg} / \mathrm{m}^{2}$ & $24.64 \pm 3.35$ & $24.40 \pm 3.34$ & $24.56 \pm 3.22$ & $24.72 \pm 3.24$ & 0.363 \\
\hline $\mathrm{HR}, \mathrm{bmp}$ & $\begin{array}{l}80.36 \pm \\
10.68\end{array}$ & $80.44 \pm 10.48$ & $80.24 \pm 11.56$ & $79.78 \pm 10.38$ & 0.719 \\
\hline SBP, mmHg & $\begin{array}{l}134.77 \pm \\
18.60\end{array}$ & $135.27 \pm 19.28$ & $135.63 \pm 18.59$ & $\begin{array}{l}135.66 \pm \\
18.32\end{array}$ & 0.830 \\
\hline $\mathrm{DBP}, \mathrm{mmHg}$ & $\begin{array}{l}82.34 \pm \\
11.16\end{array}$ & $82.20 \pm 10.96$ & $82.90 \pm 10.97$ & $82.13 \pm 11.23$ & 0.616 \\
\hline $\mathrm{PP}, \mathrm{mmHg}$ & $\begin{array}{l}52.44 \pm \\
15.24\end{array}$ & $53.07 \pm 15.88$ & $52.73 \pm 15.35$ & $53.53 \pm 15.60$ & 0.650 \\
\hline Smoker, No. (\%) & $189(31.50)$ & $157(26.48)$ & 169 (28.36) & $151(25.34)$ & 0.091 \\
\hline Drinker, No. (\%) & $145(24.17)$ & $121(20.40)$ & $132(22.15)$ & $111(18.62)$ & 0.111 \\
\hline $\begin{array}{l}\text { Antidiabetic agents, } \\
\text { yes, No. [\%] }\end{array}$ & $564(94.00)$ & $560(94.44)$ & $556(93.29)$ & 545 (91.44) & 0.174 \\
\hline $\begin{array}{l}\text { Diabetes duration, } \\
\text { year }\end{array}$ & $8.28 \pm 6.80$ & $8.62 \pm 7.06$ & $8.58 \pm 6.92$ & $8.47 \pm 7.11$ & 0.840 \\
\hline $\mathrm{HbA} 1 \mathrm{c}, \%$ & $8.62 \pm 2.49$ & $8.21 \pm 2.12$ & $8.56 \pm 2.50$ & $8.97 \pm 2.04$ & $\begin{array}{l}< \\
0.001\end{array}$ \\
\hline (continued) & & & & & \\
\hline
\end{tabular}

Relationships between quartiles of IGP and DR and its severity were shown in Table 3 with same adjusted models as above. Significant correlations between the third $(\mathrm{OR}, 1.34 ; 95 \% \mathrm{Cl}, 1.05-1.72 ; P=0.019)$ and fourth (OR, 1.30; $95 \% \mathrm{Cl}, 1.01-1.67 ; P=0.040)$ quartile of IGP and DR were obtained in Model 3.

Furthermore, among three models, every quartile of IGP was significantly related to VTDR referring to the lowest quartile (all $P$ value for trend $<0.001$ ). 
Table 3

Multivariable-adjusted associations of incremental glucose peak (IGP) and diabetic retinopathy (DR) remodeling

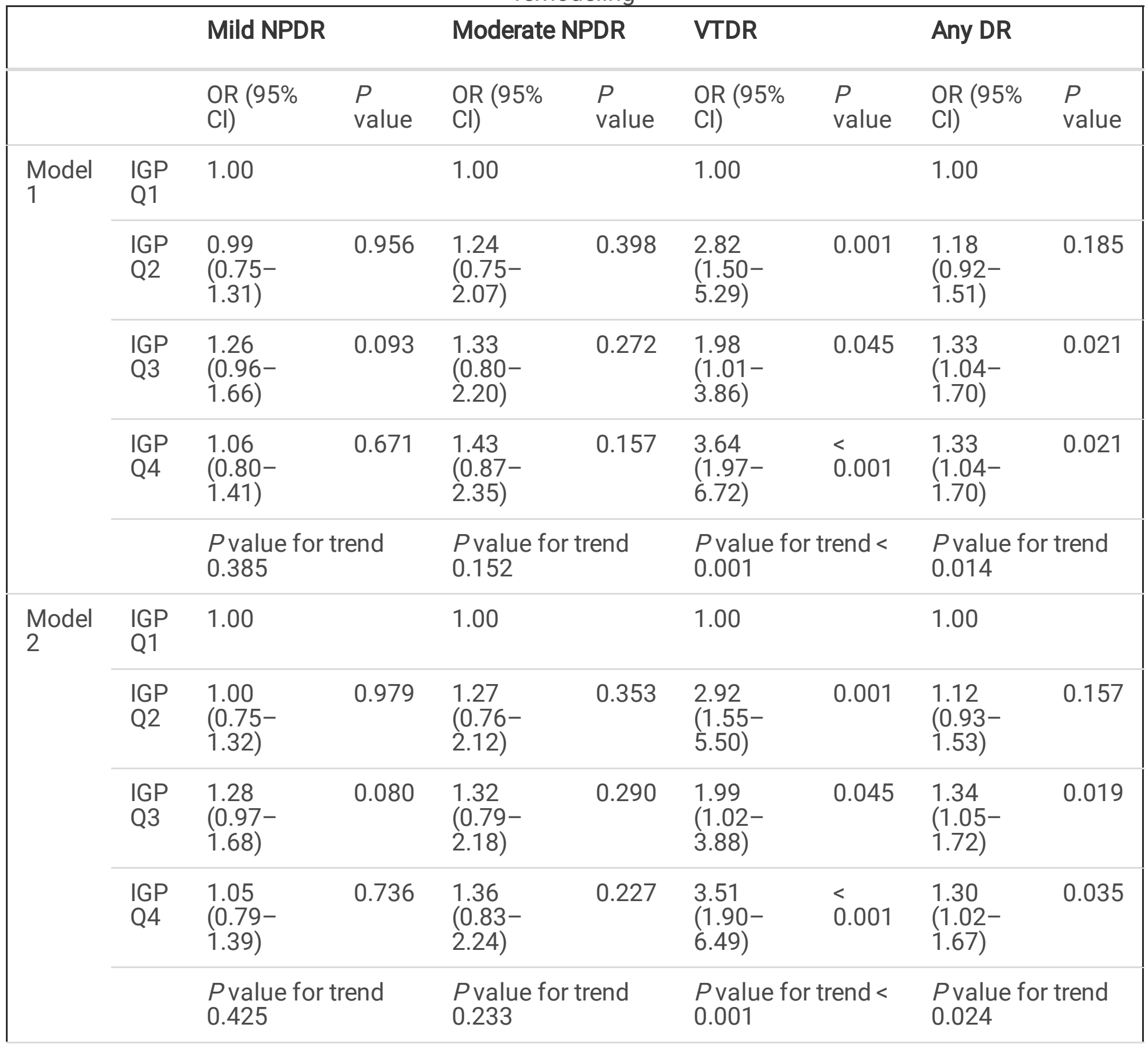

Model 1: includes gender and age;

Model 2: includes gender, age, BMI, SBP, PP, antidiabetic agents, diabetes duration, and HbA1c;

Model 3: includes gender, age, BMI, SBP, PP, antidiabetic agents, diabetes duration, HbA1c, FPG, HOMA-IR, serum creatinine, and eGFR.

Abbreviation: IGP, incremental glucose peak; DR, diabetic retinopathy; NPDR, non-proliferative diabetic retinopathy; VTDR, vision-threatening diabetic retinopathy; BMI, body mass index; SBP, systolic blood pressure; PP, pulse pressure; HbA1c, glycated hemoglobin A1c; FPG, fasting plasma glucose; HOMA-IR, the homeostasis model assessment of insulin resistance index; eGFR, estimated glomerular filtration rate; $\mathrm{OR}$, odds ratio; $\mathrm{Cl}$, confidence interval. 


\begin{tabular}{|c|c|c|c|c|c|c|c|c|c|}
\hline & \multicolumn{3}{|c|}{ Mild NPDR } & \multicolumn{2}{|c|}{ Moderate NPDR } & \multicolumn{2}{|l|}{ VTDR } & \multicolumn{2}{|c|}{ Any DR } \\
\hline \multirow[t]{5}{*}{$\begin{array}{l}\text { Model } \\
3\end{array}$} & $\begin{array}{l}\text { IGP } \\
\text { Q1 }\end{array}$ & 1.00 & & 1.00 & & 1.00 & & 1.00 & \\
\hline & $\begin{array}{l}\text { IGP } \\
\text { Q2 }\end{array}$ & $\begin{array}{l}1.00 \\
(0.75- \\
1.32)\end{array}$ & 0.975 & $\begin{array}{l}1.28 \\
(0.77- \\
2.14)\end{array}$ & 0.349 & $\begin{array}{l}3.07 \\
(1.62- \\
5.82)\end{array}$ & 0.001 & $\begin{array}{l}1.20 \\
(0.94- \\
1.54)\end{array}$ & 0.148 \\
\hline & $\begin{array}{l}\text { IGP } \\
\text { Q3 }\end{array}$ & $\begin{array}{l}1.28 \\
(0.97- \\
1.68)\end{array}$ & 0.081 & $\begin{array}{l}1.31 \\
(0.78- \\
2.18)\end{array}$ & 0.306 & $\begin{array}{l}2.05 \\
(1.04- \\
4.03)\end{array}$ & 0.038 & $\begin{array}{l}1.34 \\
(1.05- \\
1.72)\end{array}$ & 0.019 \\
\hline & $\begin{array}{l}\text { IGP } \\
\text { Q4 }\end{array}$ & $\begin{array}{l}1.04 \\
(0.78- \\
1.39)\end{array}$ & 0.774 & $\begin{array}{l}1.33 \\
(0.80- \\
2.22)\end{array}$ & 0.268 & $\begin{array}{l}3.66 \\
(1.96- \\
6.87)\end{array}$ & $<.001$ & $\begin{array}{l}1.30 \\
(1.01- \\
1.67)\end{array}$ & 0.040 \\
\hline & & $\begin{array}{l}P \text { value } \\
0.450\end{array}$ & nd & $\begin{array}{l}P \text { value } \\
0.280\end{array}$ & end & $\begin{array}{l}P \text { value } \\
0.001\end{array}$ & rend $<$ & $\begin{array}{l}P \text { value } \\
0.029\end{array}$ & trend \\
\hline \multicolumn{10}{|c|}{ Model 1: includes gender and age; } \\
\hline \multicolumn{10}{|c|}{ Model 2: includes gender, age, BMI, SBP, PP, antidiabetic agents, diabetes duration, and $\mathrm{HbA} 1 \mathrm{c}$; } \\
\hline \multicolumn{10}{|c|}{$\begin{array}{l}\text { Model 3: includes gender, age, BMI, SBP, PP, antidiabetic agents, diabetes duration, HbA1c, FPG, } \\
\text { HOMA-IR, serum creatinine, and eGFR. }\end{array}$} \\
\hline \multicolumn{10}{|c|}{$\begin{array}{l}\text { Abbreviation: IGP, incremental glucose peak; DR, diabetic retinopathy; NPDR, non-proliferative diabetic } \\
\text { retinopathy; VTDR, vision-threatening diabetic retinopathy; BMI, body mass index; SBP, systolic blood } \\
\text { pressure; PP, pulse pressure; HbA1c, glycated hemoglobin A1c; FPG, fasting plasma glucose; HOMA-IR, } \\
\text { the homeostasis model assessment of insulin resistance index; eGFR, estimated glomerular filtration } \\
\text { rate; OR, odds ratio; Cl, confidence interval. }\end{array}$} \\
\hline
\end{tabular}

\section{Additional Analyses}

Multiple R-squared liner regression including variables about DR severity, OGTT-derived parameters, HOMA-IR, and an index of renal function were used for further assessment of the association between IGP and demographic as well as biomedical variables (Table 4). After stepwise selection of variables in model 1 and 2 , only pre- and post-load index entered the models (both adjusted $R^{2}=0.908$ ) with remarkable significance (all $P<0.001$ ). 
Table 4

Associations between IGP and other variables

\begin{tabular}{|c|c|c|c|c|}
\hline \multirow[t]{2}{*}{ Variables } & \multicolumn{2}{|c|}{ Model 1} & \multicolumn{2}{|c|}{ Model 2} \\
\hline & $\boldsymbol{\beta}$ & $P$ value & $\beta$ & $P$ value \\
\hline Male & -0.006 & 0.320 & -0.017 & 0.030 \\
\hline Age, years & 0.005 & 0.464 & -0.009 & 0.297 \\
\hline $\mathrm{BMI}, \mathrm{kg} / \mathrm{m}^{2}$ & -0.002 & 0.808 & -0.002 & 0.772 \\
\hline $\mathrm{SBP}, \mathrm{mmHg}$ & -0.011 & 0.313 & -0.011 & 0.290 \\
\hline $\mathrm{PP}, \mathrm{mmHg}$ & 0.016 & 0.152 & 0.017 & 0.132 \\
\hline Antidiabetic agents, yes & -0.007 & 0.276 & -0.007 & 0.256 \\
\hline Diabetes duration, years & 0.006 & 0.354 & 0.006 & 0.376 \\
\hline $\mathrm{HbA} 1 \mathrm{c}, \%$ & -0.008 & 0.330 & -0.005 & 0.490 \\
\hline $\mathrm{FPG}, \mathrm{mmol} / \mathrm{L}$ & -1.026 & $<0.001$ & -1.025 & $<0.001$ \\
\hline Post-load glucose, mmol/L & 0.120 & $<0.001$ & 0.121 & $<0.001$ \\
\hline $30 \mathrm{~min}$ & 0.409 & $<0.001$ & 0.410 & $<0.001$ \\
\hline $60 \mathrm{~min}$ & 0.618 & $<0.001$ & 0.616 & $<0.001$ \\
\hline $120 \mathrm{~min}$ & 0.267 & $<0.001$ & 0.267 & $<0.001$ \\
\hline \multicolumn{5}{|l|}{$180 \mathrm{~min}$} \\
\hline HOMA-IR & -0.007 & 0.277 & -0.008 & 0.271 \\
\hline Serum creatinine, $\mu \mathrm{mol} / \mathrm{L}$ & & & -0.024 & 0.063 \\
\hline eGFR, $\mathrm{ml} / \mathrm{min} / 1.73 \mathrm{~m}^{2}$ & & & -0.035 & 0.021 \\
\hline
\end{tabular}

Model 1: includes gender, age, BMI, SBP, PP, antidiabetic agents, duration of diabetes, HbA1c, FPG, post-meal glucose (30 min, $60 \mathrm{~min}, 120 \mathrm{~min}$, and $180 \mathrm{~min}$ ), and HOMA-IR;

Model 2: includes gender, age, BMI, SBP, PP, antidiabetic agents, duration of diabetes, HbA1c, FPG, post-meal glucose (30 min, $60 \mathrm{~min}, 120 \mathrm{~min}$, and $180 \mathrm{~min}$ ), HOMA-IR, serum creatinine, and eGFR.

Abbreviation: IGP, incremental glucose peak; BMI, body mass index; SBP, systolic blood pressure; PP, pulse pressure; HbA1c, glycated hemoglobin A1c; FPG, fasting plasma glucose; HOMA-IR, the homeostasis model assessment of insulin resistance index; eGFR, estimated glomerular filtration rate.

* Using stepwise method to select variables. Stepwise method ordinarily included the most significant variable $(P<0.05)$ and then excluded the most indistinctive variable $(P>0.10)$, which was aimed to obtain an optimal combine of variables based on Forward method and Backward method. 


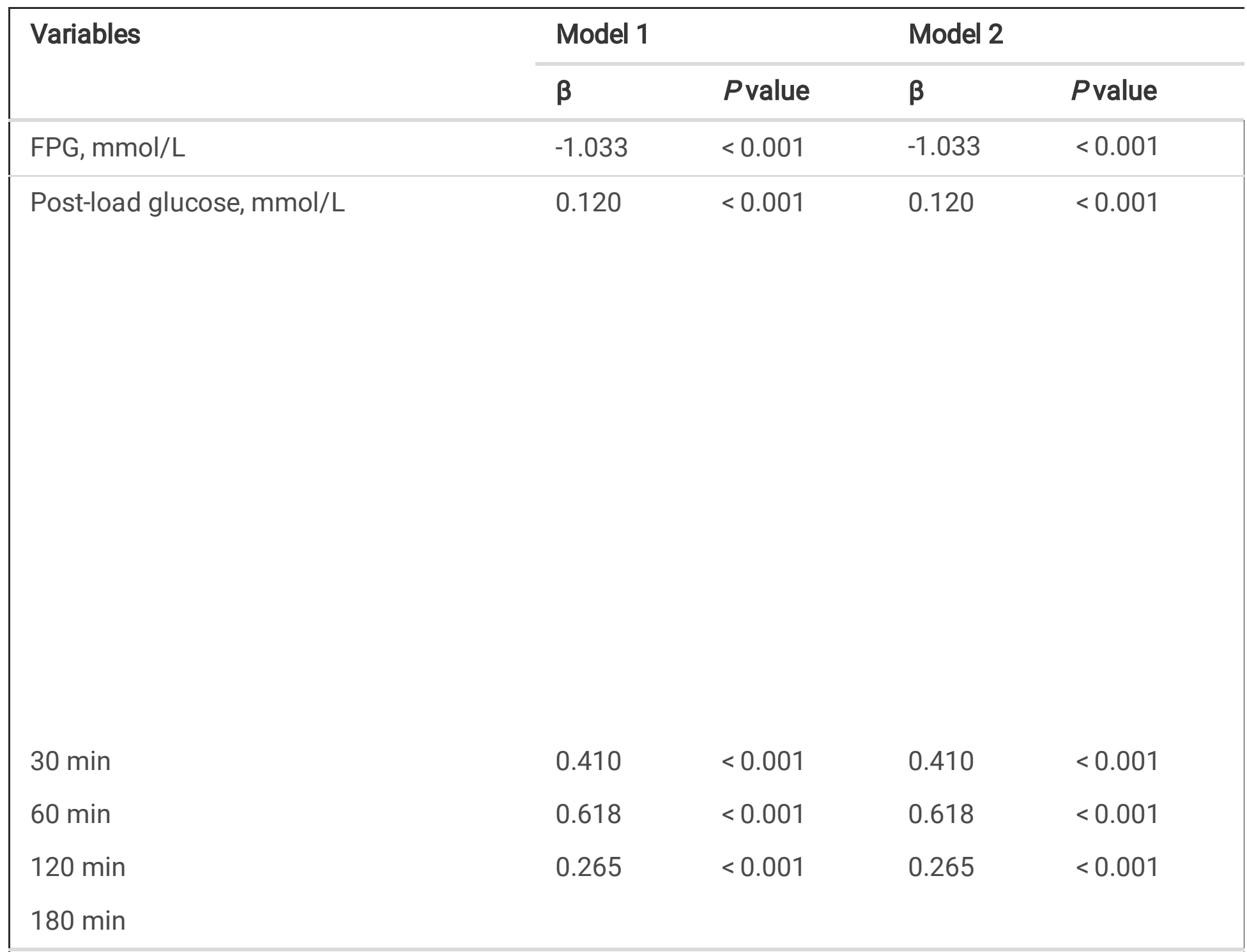

Model 1: includes gender, age, BMI, SBP, PP, antidiabetic agents, duration of diabetes, HbA1c, FPG, post-meal glucose (30 min, $60 \mathrm{~min}, 120 \mathrm{~min}$, and $180 \mathrm{~min}$ ), and HOMA-IR;

Model 2: includes gender, age, BMI, SBP, PP, antidiabetic agents, duration of diabetes, HbA1c, FPG, post-meal glucose (30 min, $60 \mathrm{~min}, 120 \mathrm{~min}$, and $180 \mathrm{~min}$ ), HOMA-IR, serum creatinine, and eGFR.

Abbreviation: IGP, incremental glucose peak; BMI, body mass index; SBP, systolic blood pressure; PP, pulse pressure; HbA1c, glycated hemoglobin A1c; FPG, fasting plasma glucose; HOMA-IR, the homeostasis model assessment of insulin resistance index; eGFR, estimated glomerular filtration rate.

* Using stepwise method to select variables. Stepwise method ordinarily included the most significant variable $(P<0.05)$ and then excluded the most indistinctive variable $(P>0.10)$, which was aimed to obtain an optimal combine of variables based on Forward method and Backward method.

\section{Discussion}

In current study, we investigated case-control associations of IGP with DR as well as its severity in Chinese participants with type 2 diabetes. Firstly, our findings revealed the relationships between OGTTderived indicators and the risk of retinopathy. $\mathrm{HbA} 1 \mathrm{c}$ and 120-min PG value has previously been found to be independently associated with DR which conformed with our findings,[7, 22] while the evidence on the 
association between 180-min PG value and DR were limited which might partly depend on 120-min PG. Post-load plasma glucose peak, which was described as a risk factor of cardiovascular diseases with postprandial peak,[23] also had impact of hyperglycaemia on endothelial function and retinal vascular reactivity. Previous studies had proposed that FPG could screen high risk diabetes with microvascular events and 60-min PG could prompt the onset of DR due to theirs close connection with insulin secretion. $[22,24]$ By contrast, in our study no independent association between FPG and 60-min OGTT glucose concentration and presence of DR was found. However, their use of cross-sectional design and 1- or 2-h plasma glucose concentrations by Americans or Indians entails a major different finding.

Additionally, adjusting for gender, age, and potential clinical-based confounders for DR, this present study first reported the association of IGP index with the presence of any-DR, moderate NPDR, and VTDR. This finding was also consistent with the results when IGP level was considered into categorical variable. Although there is no direct evidence on IGP levels with DR, high IGP was found to be associated with several macrovascular impairments, such as aortic stiffness, carotid intima-media thickness (CIMT), and maladaptive carotid remodeling.[16, 17] Moreover, evidences showed that when CGM was unavailable, IGP could be chosen to assess GV in replacement of coefficient of variation (CV; SD / mean * 100\%),[14] which was recommended as the leading measurement of variability by the international consensus of the Advanced Technologies \& Treatments for Diabetes (ATTD) Congress.[25] The association between variability assessed by IGP and retinopathy was consistent with the results of the GV metrics assessed by CGM, $[11,13]$ while the results of some researches remained in debate and further longitudinal, population-based studies are needed to confirm our findings .[10]

\section{Mechanistic Explanations}

The biological pathway underlying the relationship between IGP and DR remains to be elucidated. In order to fill this gap, HOMA-IR which was associated with DR, was graded by quartiles, and higher level of insulin resistance increased FPG and 3-h post-load plasma glucose peak (both $P$ value $<0.001$ ), but decreased the level of IGP (see Figure S2 in Additional file 3). In contrast, Murata et al. got a positive correlation between glucose fluctuation and HOMA-IR due to definitional discrepancy between glucose fluctuation and IPG.[26] In our study, IGP could reflect an integrated situation of pre-load and 3-h postload plasma glucose and seem to be related to insulin resistance, then form a relationship with DR.

In addition, DR has been traditionally recognized as a micro -vascular disease. Nevertheless, retinal neurodegeneration (neuronal apoptosis and glial activation) was also proved to be involved in the pathogenesis of DR.[27] The activation of Müller cells, the representative glial component of retina, was observed in response to GV.[28] Besides, Picconi et al. and Stem et al. demonstrated that increased glycemic excursion was connected with early structural impairments of neural retina in patients with type 1 diabetes, resulting a stable glycemic status and a decrease in GV might prevent the damage to structure and function of retina.[29, 30] 
Although molecular mechanisms induced by hyperglycemia in the incidence and progression of DR have been described in previous studies, such as the hexosamine pathway, increased polyol pathway flux, protein kinase $\mathrm{C}$ (PKC) activation, free radicals formation and advanced glycation end (AGE) products accumulation, the metabolic mechanism of GV was unclear yet.[9] Some evidences indicated that oscillating glucose played a more destructive role on oxidative stress and endothelial function than sustained hyperglycemia.[31, 32] And a further mechanism revealed oscillating glucose left a metabolic memory, which suggested the persistence of intracellular pro-oxidant environment after plasma glucose normalization.[33] More observational and experimental evidence for the effect of GV on retinopathy was expected.

\section{Clinical Relevance}

Diabetes mellitus causes some macrovascular complications such as cardiovascular diseases (CVD) as well as microvascular complications such as retinopathy, nephropathy, and neuropathy, of which, DR is a major cause of vision loss in middle-aged and elderly people. Previous study reported that IPG was associated with aortic stiffness, which is an independent determinant of CVD. Currently, we found that after adjustment for relevant confounders risk of DR was increased with IGP increasing. Our results may imply that, even in case of well-controlled obesity, blood pressure, HbA1c, and renal function, the harmful effects of IPG on DR are still present. Future studies should investigate whether our findings translate to other microvascular complications. If they are replicated in nephropathy, and neuropathy, it would further justify therapeutic interventions that specifically target IGP.

\section{Strengths And Limitations}

In current study, a relative large sample size, standardized information collection, and a comprehensive mixed model analysis strengthened the reliability of our findings. However, some limitations should be noted. Firstly, the random measurement error might be induced by IGP, which was calculated by the indices derived from the 3-h OGTT with moderate reproducibility. Secondly, GV was an integrated definition by amplitude, frequency, and duration of the excursions,[25] but IGP merely emphasized the amplitude as a substitution of professional CGM. Thirdly, individuals this study only included Chinese hospitalized adults, which restricted the generalizability of the outcomes. Fourthly, a relatively large number of subjects were excluded due to uncompleted OGTT data. Fifth, the cross-sectional design renders us unable to rule out reverse causality.

\section{Conclusions}

In brief, the present study showed that 3-h OGTT-derived IGP was significantly correlated with the onset and progression of DR in in Chinese patients with type 2 diabetes. This finding suggested that glycemic control of DR patients should be additionally targeted at IPG to approach the normally glycemic 
condition. Future research is needed to elucidate the effect of IGP on DR and to what extent DR could be prevented by reducing IPG.

\section{Abbreviations}

IGP, incremental glucose peak; DR, diabetic retinopathy; LD-MCS, Liaoning Diabetic Microvascular Complications Study; ADA, American Diabetes Association; CMU1h, the First Affiliated Hospital of China Medical University; OGTT, oral glucose tolerance test; NPDR, non-proliferative diabetic retinopathy; VTDR, vision-threatening diabetic retinopathy; UKPDS, United Kingdom Prospective Diabetes Study; DCCT, Diabetes Control and Complications Trial; HbA1c, glycated hemoglobin A1c; GV, glycemic variability; SD, standard deviation; MAGE, mean amplitude of glycemic excursions; CGM, continuous glucose monitoring system; LD-RS, Liaoning Diabetic Retinopathy Study; LD-CKDS, Liaoning Diabetic Chronic Kidney Disease Study; LD-PNS, Liaoning Diabetic Peripheral Neuropathy Study; IRB, Institutional Review Board; ETDRS, Early Treatment for Diabetic Retinopathy Study; PDR, proliferative diabetic retinopathy; HR, heart rate; SBP, systolic blood pressure; DBP, diastolic blood pressure; PP, pulse pressure; FPG, fasting plasma glucose; $P G$, plasma glucose; HOMA-IR, model assessment of insulin resistance index; HOMA- $\beta$, homeostasis model assessment of $\beta$-cell function; eGFR, estimated glomerular filtration rate; CKD-EPI, Chronic Kidney Disease Epidemiology Collaboration; $\mathrm{OR}$, odds ratio; $\mathrm{Cl}$, confidence interval; Q, quartile; $\mathrm{CIMT}$, carotid intima-media thickness; CV, coefficient of variation; ATTD, Advanced Technologies \& Treatments for Diabetes; PKC, protein kinase C; AGE, advanced glycation end.

\section{Declarations}

Ethics approval and consent to participate The study adhered to the content detailed in the Declaration of Helsinki and got approval from the Institutional Review Board (IRB) of the First Affiliated Hospital of China Medical University (AF-SOP-07-1.1-01/2019-13).

Consent for publication Not applicable.

Availability of data and materials The datasets used and/or analysed during the current study are available from the corresponding author on reasonable request.

Competing interests No conflicting relationship exists for any author.

Funding This article is supported by the National Natural Science Foundation of China (No. 81300783), China Postdoctoral Science Foundation (No. 2019TQ0358; No. 2019M661162), and Liaoning Revitalization Talents Program (No. XLYC1807082). The funders had no involvement in the study design, data collection and analysis, decision to publish, or preparation of the manuscript.

Authors' contributions Contributed equally to this work

Acknowledgements We are particularly grateful to the involvement and contribution of study participants in our research. Thanks to Shenyang Young and Middle-aged Science and Technology Innovation Talent 
Support Program (RC190146) and other researchers in the Department of Endocrinology and Metabolism, First Hospital of China Medical University.

Authors' information Not applicable.

\section{References}

1. Ting DS, Cheung GC, Wong TY. Diabetic retinopathy: global prevalence, major risk factors, screening practices and public health challenges: a review. Clin Exp Ophthalmol. 2016;44(4):260-77.

2. Wong TY, Cheung CM, Larsen M, Sharma S, Simó R. Diabetic retinopathy. Nat Rev Dis Primers. 2016;2:16012.

3. Yau JW, Rogers SL, Kawasaki R, Lamoureux EL, Kowalski JW, Bek T, et al. Global prevalence and major risk factors of diabetic retinopathy. Diabetes Care. 2012;35(3):556-64.

4. Sabanayagam C, Banu R, Chee ML, Lee R, Wang YX, Tan G, et al. Incidence and progression of diabetic retinopathy: a systematic review. The Lancet Diabetes Endocrinology. 2019;7(2):140-9.

5. Trento M, Passera P, Trevisan M, Schellino F, Sitia E, Albani S, et al. Quality of life, impaired vision and social role in people with diabetes: a multicenter observational study. Acta Diabetol. 2013;50(6):8737.

6. Mohamed Q, Gillies MC, Wong TY. Management of diabetic retinopathy: a systematic review. Jama. 2007;298(8):902-16.

7. Group UKPDS. Intensive blood-glucose control with sulphonylureas or insulin compared with conventional treatment and risk of complications in patients with type 2 diabetes (UKPDS 33). UK Prospective Diabetes Study (UKPDS) Group. Lancet. 1998;352(9131):837-53.

8. Nathan DM, Genuth S, Lachin J, Cleary P, Crofford O, Davis M, et al. The effect of intensive treatment of diabetes on the development and progression of long-term complications in insulin-dependent diabetes mellitus. N Engl J Med. 1993;329(14):977-86.

9. Safi SZ, Qvist R, Kumar S, Batumalaie K, Ismail IS. Molecular mechanisms of diabetic retinopathy, general preventive strategies, and novel therapeutic targets. BioMed research international. 2014;2014:801269.

10. Sonoda S, Okada Y, Mori H, Uemura F, Sugai K, Hajime M, et al. Association Between Diabetic Microangiopathies and Glycemic Variability Assessed by Continuous Glucose Monitoring. J UOEH. 2018;40(1):11-8.

11. Sartore G, Chilelli NC, Burlina S, Lapolla A. Association between glucose variability as assessed by continuous glucose monitoring (CGM) and diabetic retinopathy in type 1 and type 2 diabetes. Acta Diabetol. 2013;50(3):437-42.

12. Selvin E, Rawlings AM, Grams M, Klein R, Steffes M, Coresh J. Association of 1,5-anhydroglucitol with diabetes and microvascular conditions. Clin Chem. 2014;60(11):1409-18.

13. Lu J, Ma X, Zhou J, Zhang L, Mo Y, Ying L, et al. Association of Time in Range, as Assessed by Continuous Glucose Monitoring, With Diabetic Retinopathy in Type 2 Diabetes. Diabetes Care. 
2018;41(11):2370-6.

14. Foreman YD, Brouwers M, van der Kallen CJH, Pagen DME, van Greevenbroek MMJ, Henry RMA, et al. Glucose Variability Assessed with Continuous Glucose Monitoring: Reliability, Reference Values, and Correlations with Established Glycemic Indices-The Maastricht Study. Diabetes technology \& therapeutics. 2020.

15. Meier JJ, Baller B, Menge BA, Gallwitz B, Schmidt WE, Nauck MA. Excess glycaemic excursions after an oral glucose tolerance test compared with a mixed meal challenge and self-measured home glucose profiles: is the OGTT a valid predictor of postprandial hyperglycaemia and vice versa? Diabetes Obes Metab. 2009;11(3):213-22.

16. Foreman YD, Brouwers $M$, Berendschot $T$, van Dongen $M$, Eussen $S$, van Greevenbroek MMJ, et al. The oral glucose tolerance test-derived incremental glucose peak is associated with greater arterial stiffness and maladaptive arterial remodeling: The Maastricht Study. Cardiovasc Diabetol. 2019;18(1):152.

17. Esposito K, Ciotola M, Carleo D, Schisano B, Sardelli L, Di Tommaso D, et al. Post-meal glucose peaks at home associate with carotid intima-media thickness in type 2 diabetes. J Clin Endocrinol Metab. 2008;93(4):1345-50.

18. Group ETDRSR. Grading diabetic retinopathy from stereoscopic color fundus photographs-an extension of the modified Airlie House classification. ETDRS report number 10. Early Treatment Diabetic Retinopathy Study Research Group. Ophthalmology. 1991;98(5 Suppl):786-806.

19. Wilkinson CP, Ferris FL 3rd, Klein RE, Lee PP, Agardh CD, Davis M, et al. Proposed international clinical diabetic retinopathy and diabetic macular edema disease severity scales. Ophthalmology. 2003;110(9):1677-82.

20. Matthews DR, Hosker JP, Rudenski AS, Naylor BA, Treacher DF, Turner RC. Homeostasis model assessment: insulin resistance and beta-cell function from fasting plasma glucose and insulin concentrations in man. Diabetologia. 1985;28(7):412-9.

21. Levey AS, Stevens LA, Schmid CH, Zhang YL, Castro AF 3rd, Feldman HI, et al. A new equation to estimate glomerular filtration rate. Ann Intern Med. 2009;150(9):604-12.

22. Gabir MM, Hanson RL, Dabelea D, Imperatore G, Roumain J, Bennett PH, et al. Plasma glucose and prediction of microvascular disease and mortality: evaluation of 1997 American Diabetes Association and 1999 World Health Organization criteria for diagnosis of diabetes. Diabetes Care. 2000;23(8):1113-8.

23. Bonora E. Postprandial peaks as a risk factor for cardiovascular disease: epidemiological perspectives. International journal of clinical practice Supplement. 2002(129):5-11.

24. Paddock E, Looker HC, Piaggi P, Knowler WC, Krakoff J, Chang DC. One-Hour Plasma Glucose Compared With Two-Hour Plasma Glucose in Relation to Diabetic Retinopathy in American Indians. Diabetes Care. 2018;41(6):1212-7.

25. Danne T, Nimri R, Battelino T, Bergenstal RM, Close KL, DeVries JH, et al. International Consensus on Use of Continuous Glucose Monitoring. Diabetes Care. 2017;40(12):1631-40. 
26. Murata M, Adachi H, Oshima S, Kurabayashi M. Glucose fluctuation and the resultant endothelial injury are correlated with pancreatic $\beta$ cell dysfunction in patients with coronary artery disease. Diabetes Res Clin Pract. 2017;131:107-15.

27. Simó R, Hernández C. Neurodegeneration is an early event in diabetic retinopathy: therapeutic implications. Br J Ophthalmol. 2012;96(10):1285-90.

28. Picconi F, Parravano M, Sciarretta F, Fulci C, Nali M, Frontoni S, et al. Activation of retinal Müller cells in response to glucose variability. Endocrine. 2019;65(3):542-9.

29. Picconi F, Parravano M, Ylli D, Pasqualetti P, Coluzzi S, Giordani I, et al. Retinal neurodegeneration in patients with type 1 diabetes mellitus: the role of glycemic variability. Acta Diabetol. 2017;54(5):48997.

30. Stem MS, Dunbar GE, Jackson GR, Farsiu S, Pop-Busui R, Gardner TW. Glucose variability and inner retinal sensory neuropathy in persons with type 1 diabetes mellitus. Eye. 2016;30(6):825-32.

31. Ceriello A, Esposito K, Piconi L, Ihnat MA, Thorpe JE, Testa R, et al. Oscillating glucose is more deleterious to endothelial function and oxidative stress than mean glucose in normal and type 2 diabetic patients. Diabetes. 2008;57(5):1349-54.

32. Monnier L, Mas E, Ginet C, Michel F, Villon L, Cristol JP, et al. Activation of oxidative stress by acute glucose fluctuations compared with sustained chronic hyperglycemia in patients with type 2 diabetes. Jama. 2006;295(14):1681-7.

33. Schisano B, Tripathi G, McGee K, McTernan PG, Ceriello A. Glucose oscillations, more than constant high glucose, induce p53 activation and a metabolic memory in human endothelial cells. Diabetologia. 2011;54(5):1219-26.

\section{Figures}




\begin{tabular}{|c|c|c|c|}
\hline & OR $(95 \%$ CI $)$ & OR $(95 \% \mathrm{CI})$ & $P$ value \\
\hline \multicolumn{4}{|l|}{ Any DR } \\
\hline Model 1 & $\mapsto$ & $1.05(1.02-1.07)$ & $<0.001$ \\
\hline Model 2 & $\mapsto$ 멉 & $1.05(1.02-1.07)$ & 0.001 \\
\hline Model 3 & $\mapsto$ 밥 & $1.05(1.02-1.07)$ & 0.001 \\
\hline \multicolumn{4}{|c|}{ Mild NPDR } \\
\hline Model 1 & -1 & $1.03(1.00-1.06)$ & 0.084 \\
\hline Model 2 & -1 & $1.03(1.00-1.06)$ & 0.094 \\
\hline Model 3 & -1 & $1.03(1.00-1.06)$ & 0.098 \\
\hline \multicolumn{4}{|c|}{ Moderate NPDR } \\
\hline Model 1 & $\longmapsto$ & $1.06(1.01-1.12)$ & 0.020 \\
\hline Model 2 & $\longmapsto$ & $1.06(1.01-1.11)$ & 0.032 \\
\hline Model 3 & $\longrightarrow$ & $1.06(1.00-1.11)$ & 0.041 \\
\hline \multicolumn{4}{|l|}{ VTDR } \\
\hline Model 1 & $\longmapsto \longrightarrow$ & $1.13(1.07-1.19)$ & $<0.001$ \\
\hline Model 2 & $\longmapsto$ & $1.12(1.07-1.18)$ & $<0.001$ \\
\hline Model 3 & $\longmapsto$ & $1.13(1.07-1.19)$ & $<0.001$ \\
\hline \multicolumn{4}{|c|}{0.9511 .051 .11 .151 .2} \\
\hline
\end{tabular}

\section{Figure 1}

Associations between incremental glucose peak (IGP) and stages of DR after controlling for confounders Model 1: includes gender and age; Model 2: includes gender, age, BMI, SBP, PP, antidiabetic agents, diabetes duration, and $\mathrm{HbA1c}$; Model 3: includes gender, age, BMI, SBP, PP, antidiabetic agents, diabetes duration, HbA1c, FPG, HOMA-IR, serum creatinine, and eGFR. Abbreviation: IGP, incremental glucose peak; DR, diabetic retinopathy; NPDR, non-proliferative diabetic retinopathy; VTDR, vision-threatening diabetic retinopathy; $\mathrm{BMI}$, body mass index; SBP, systolic blood pressure; $\mathrm{PP}$, pulse pressure; HbA1c, glycated hemoglobin A1c; FPG, fasting plasma glucose; HOMA-IR, the homeostasis model assessment of insulin resistance index; eGFR, estimated glomerular filtration rate; $\mathrm{OR}$, odds ratio; $\mathrm{Cl}$, confidence interval. Error bars represent the $95 \%$ confidence interval of odd ratios in IGP. 


\begin{tabular}{|c|c|c|c|}
\hline & OR $(95 \% \mathrm{CI})$ & OR $(95 \%$ CI $)$ & $P$ value \\
\hline \multicolumn{4}{|l|}{ Any DR } \\
\hline Model 1 & $\mathrm{P}^{\mapsto}$ & $1.05(1.02-1.07)$ & $<0.001$ \\
\hline Model 2 & $\mapsto$ 마 & $1.05(1.02-1.07)$ & 0.001 \\
\hline Model 3 & $\mapsto$ 밤 & $1.05(1.02-1.07)$ & 0.001 \\
\hline \multicolumn{4}{|c|}{ Mild NPDR } \\
\hline Model 1 & -1 & $1.03(1.00-1.06)$ & 0.084 \\
\hline Model 2 & -1 & $1.03(1.00-1.06)$ & 0.094 \\
\hline Model 3 & -1 & $1.03(1.00-1.06)$ & 0.098 \\
\hline \multicolumn{4}{|c|}{ Moderate NPDR } \\
\hline Model 1 & $\longmapsto-1$ & $1.06(1.01-1.12)$ & 0.020 \\
\hline Model 2 & $\longmapsto$ & $1.06(1.01-1.11)$ & 0.032 \\
\hline Model 3 & $\longrightarrow$ & $1.06(1.00-1.11)$ & 0.041 \\
\hline \multicolumn{4}{|l|}{ VTDR } \\
\hline Model 1 & $\longmapsto \longrightarrow$ & $1.13(1.07-1.19)$ & $<0.001$ \\
\hline Model 2 & $\longmapsto-1$ & $1.12(1.07-1.18)$ & $<0.001$ \\
\hline Model 3 & $\longmapsto$ & $1.13(1.07-1.19)$ & $<0.001$ \\
\hline & 0.9511 .051 .11 .151 & & \\
\hline
\end{tabular}

\section{Figure 1}

Associations between incremental glucose peak (IGP) and stages of DR after controlling for confounders Model 1: includes gender and age; Model 2: includes gender, age, BMI, SBP, PP, antidiabetic agents, diabetes duration, and $\mathrm{HbA1c}$; Model 3: includes gender, age, BMI, SBP, PP, antidiabetic agents, diabetes duration, HbA1c, FPG, HOMA-IR, serum creatinine, and eGFR. Abbreviation: IGP, incremental glucose peak; DR, diabetic retinopathy; NPDR, non-proliferative diabetic retinopathy; VTDR, vision-threatening diabetic retinopathy; $\mathrm{BMI}$, body mass index; $\mathrm{SBP}$, systolic blood pressure; $\mathrm{PP}$, pulse pressure; $\mathrm{HbA1c}$, glycated hemoglobin A1c; FPG, fasting plasma glucose; HOMA-IR, the homeostasis model assessment of insulin resistance index; eGFR, estimated glomerular filtration rate; $\mathrm{OR}$, odds ratio; $\mathrm{Cl}$, confidence interval. Error bars represent the $95 \%$ confidence interval of odd ratios in IGP.

\section{Supplementary Files}

This is a list of supplementary files associated with this preprint. Click to download.

- Additionalfile1.pdf

- Additionalfile1.pdf

- Additionalfile2.pdf

- Additionalfile2.pdf 
- Additonalfile3.pdf

- Additonalfile3.pdf 